\title{
Lymph node involvement in esophageal adenocarcinoma: If you see one, have you seen them all?
}

Steven R. DeMeester, MD

See related article on page 1121.

From the Department of Cardiothoracic Surgery, Keck School of Medicine, the University of Southern California, Los Angeles, Calif.

Received for publication April 1, 2003; accepted for publication April 15, 2003.

Address for reprints: Steven R. DeMeester, MD, Assistant Professor of Cardiothoracic Surgery, Norris Comprehensive Cancer Center, 1441 Eastlake Ave, Suite 7418, Los Angeles, CA 90033-0804 (E-mail: sdemeester@surgery.usc.edu).

J Thorac Cardiovasc Surg 2003;126:947-9

Copyright (๑) 2003 by The American Association for Thoracic Surgery

$0022-5223 / 2003 \$ 30.00+0$

doi:10.1016/S0022-5223(03)00732-3

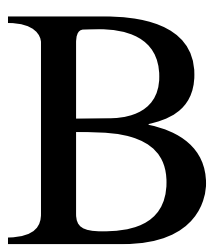

arely 50 years ago, the very existence of a primary adenocarcinoma of the esophagus was debated, and this rare tumor was a novelty in comparison with squamous cell esophageal cancer. Yet in one of the most dramatic epidemiologic transformations ever recorded, adenocarcinoma has become the predominant form of esophageal cancer, and the incidence of this cancer continues to increase in all age groups in the United States. ${ }^{1}$ Although both squamous cell carcinoma and adenocarcinoma of the esophagus are deadly diseases, the existence of a precursor lesion (Barrett) for esophageal adenocarcinoma has allowed significant advances in our understanding of the pathogenesis and natural history of this cancer. Increasingly, early adenocarcinoma is detected either within a surveillance program for Barrett or fortuitously during an endoscopy to evaluate symptoms of reflux. Many of these patients have superficial tumors and are cured with surgical resection alone, disproving previously held concepts that esophageal cancer is systemic and essentially incurable in all patients at the time of diagnosis.

The increasing incidence of esophageal adenocarcinoma and the fact that many of these patients are otherwise healthy permits an accurate assessment of the natural history of the disease and the effect of specific therapies on the course of the illness. Experience at several centers has demonstrated that one of the most important predictors of both survival and the risk of systemic disease in patients with esophageal adenocarcinoma is the presence and extent of involved lymph nodes. ${ }^{2-5}$ Although the current American Joint Committee on Cancer Staging system for esophageal carcinoma only classifies disease as N0 (no involved nodes) or N1 (1 or more involved nodes), distinct survival curves can be produced on the basis of the number, as well as the ratio, of involved to total resected lymph nodes. ${ }^{6,7}$ The depth of tumor invasion is also important, but most of the importance relates to the direct correlation between the depth of invasion and the prevalence of nodal metastases.

In this issue of the Journal, Lerut and colleagues ${ }^{8}$ examine the influence of lymph node involvement, and in particular extracapsular tumor extension, on the survival of patients undergoing primary surgical resection for adenocarcinoma of the esophagus. All patients in this retrospective study had T3 tumors and had undergone an R0 resection without neoadjuvant therapy. Similar to data from other centers, $83 \%$ of these patients with transmural tumors had one or more involved lymph glands, as determined by histologic examination. ${ }^{2,9}$ Importantly, that means that $17 \%$ of patients with locally advanced tumors did not have any lymph node metastases, and the 57\% 5-year survival in these patients was significantly better than that of patients who had lymph node metastases. ${ }^{8}$ We also found that $16 \%$ of patients with transmural tumors had no involved lymph nodes, and the 5-year survival for this group of patients after en bloc resection alone was $85 \%$ in our experience. $^{2}$

An interesting aside is that the percentage of patients with locally advanced tumors without nodal involvement is similar to the percentage of patients who are found to have a complete pathologic response after neoadjuvant chemoradiotherapy. Furthermore, the survival in this group is quite similar to the survival reported for the subgroup of patients with a complete pathologic response in several randomized trials comparing neoadjuvant chemoradiotherapy plus surgical intervention with surgical intervention alone. ${ }^{10-12}$ Recently, Jiao and associates ${ }^{13}$ have shown that 
patients with lymph node metastases proved by minimally invasive staging techniques are less likely to have a complete pathologic response to neoadjuvant therapy. Putting this information together, one might speculate that the subgroup of patients with a complete pathologic response and improved survival in the randomized trials of neoadjuvant therapy might well be those without lymph node metastases, who would have done well with surgical resection alone. Thus, pathologic pretreatment staging should be encouraged in future trials, and potentially misleading subgroup analyses should be avoided.

Lerut and colleagues ${ }^{8}$ also confirmed what has been demonstrated in other centers in regard to the importance of the number of involved lymph nodes. ${ }^{3,7}$ They found that the number of positive lymph nodes exhibited a linear effect in the Cox regression analysis of survival, with increasingly worse survival as the number of involved nodes increased up to a maximum of 12. In addition to the importance of the number of positive nodes, the characteristics of lymph nodes containing metastatic deposits might also affect survival. Recently, Dhar and coworkers ${ }^{5}$ reported that the size of involved lymph nodes influences survival in patients with esophageal cancer, and now Lerut and colleagues ${ }^{8}$ demonstrate that extracapsular tumor extension within an involved lymph node is also an important survival determinant. In the study by Lerut and colleagues, patients with extracapsular nodal involvement had a significantly worse survival than that seen in those without nodal involvement or only intracapsular nodal involvement. The most compelling argument in support of the importance of extracapsular nodal involvement was the finding that if patients with only a single positive lymph node were compared, the 5-year survival in those with extracapsular tumor extension in the positive node was significantly worse (33.3\%) than it was in those with only intracapsular nodal involvement (85.7\%). This would suggest that it is not merely that patients with extracapsular nodal involvement have more advanced disease and more involved nodes than patients with intracapsular nodal extension. Rather, it supports the argument that Lerut and colleagues ${ }^{8}$ put forth: that extracapsular nodal involvement is an independent negative prognostic factor in these patients.

Surprisingly, Lerut and colleagues ${ }^{8}$ found that survival in patients with only intracapsular nodal involvement was not significantly different from that in patients with no involved lymph nodes ( $40.9 \%$ vs $57 \%$ at 5 years, respectively). The number of patients at risk at each time interval is not provided in the Kaplan-Meier survival plot, but it is likely that this represents a type II error. This finding also suggests that most patients with intracapsular nodal involvement had only a few involved nodes; otherwise one would certainly expect there to be a significant survival difference compared with that seen in patients with no involved lymph nodes. However, because the authors do not provide information regarding the mean and median number of involved nodes for patients with intracapsular versus extracapsular nodal involvement, it is not possible to evaluate this further.

It is also difficult to explain the finding that the 5-year survival in 14 patients with 1 positive lymph node and intracapsular tumor involvement was $85.7 \% .^{8}$ This survival is much greater than the $57 \%$-year survival in patients with no involved nodes and is incongruent with the significant survival difference between patients with $\mathrm{pN} 0$ and pN1 disease reported by multiple authors, as well as the findings by some investigators that even micrometastases detected with immunohistochemical staining adversely affect survival in patients with esophageal cancer. ${ }^{14,15}$ This might again reflect a type II error because only 14 patients had isolated intracapsular nodal involvement.

Thus, although the concept of extracapsular versus intracapsular nodal involvement is intriguing and might allow further stratification of survival in patients with esophageal cancer and involved lymph nodes, the fact remains that the current staging system fails to take into account the clear importance of the number of involved nodes. Once revisions in the staging system have been accomplished that address the survival differences on the basis of the number of involved nodes, perhaps further stratification can be made on the basis of anatomic and histologic characteristics, such as the presence of capsular invasion or micrometastatic disease in lymph nodes. Ultimately, it is likely that many of these relatively crude factors will be replaced or supplemented by tumor-specific panels of biologic and molecular staging markers. In the meantime, physicians involved in the treatment of patients with esophageal cancer should pay attention to both the presence and the number of histologically involved lymph nodes.

\section{References}

1. El-Serag HB, Mason AC, Petersen N, Key CR. Epidemiological differences between adenocarcinoma of the oesophagus and adenocarcinoma of the gastric cardia in the USA. Gut. 2002;50:368-72.

2. Nigro JJ, DeMeester SR, Hagen JA, et al. Node status in transmural esophageal adenocarcinoma and outcome after en bloc esophagectomy. J Thorac Cardiovasc Surg. 1999;117:960-8.

3. Korst RJ, Rusch VW, Venkatraman E, et al. Proposed revision of the staging classification for esophageal cancer. J Thorac Cardiovasc Surg. 1998;115:660-70.

4. Altorki NMD, Skinner DMD. Should en bloc esophagectomy be the standard of care for esophageal carcinoma? Ann Surg. 2001;234:581-7.

5. Dhar DK, Tachibana M, Kinukawa N, et al. The prognostic significance of lymph node size in patients with squamous esophageal cancer. Ann Surg Oncol. 2002;9:1010-6.

6. Roder JD, Busch R, Stein HJ, et al. Ratio of invaded to removed lymph nodes as a predictor of survival in squamous cell carcinoma of the oesophagus. Br J Surg. 1994;81:410-3.

7. Hagen J, DeMeester S, Peters J, et al. Curative resection for esophageal adenocarcinoma: analysis of 100 en bloc esophagectomies. Ann Surg. 2001;234:520-31.

8. Lerut T, Coosemans W, Decker G, De Leyn P, Ectors N, Fieuws S, et al. Extracapsular lymph node involvement is a negative prognostic factor in T3 adenocarcinoma of the distal esophagus and gastroesophageal junction. J Thorac Cardiovasc Surg. 2003;126:1121-8. 
9. Rice TW, Zuccaro G Jr, Adelstein DJ, et al. Esophageal carcinoma: depth of tumor invasion is predictive of regional lymph node status. Ann Thorac Surg. 1998;65:787-92.

10. Bates BA, Detterbeck FC, Bernard SA, et al. Concurrent radiation therapy and chemotherapy followed by esophagectomy for localized esophageal carcinoma. J Clin Oncol. 1996;14:156-63.

11. Apinop C, Puttisak P, Preecha N. A prospective study of combined therapy in esophageal cancer. Hepatogastroenterology. 1994;41:391-3.

12. Urba S, Orringer M, Turisi A, et al. Randomized trial of preoperative chemoradiation versus surgery alone in patients with locoregional esophageal carcinoma. J Clin Oncol. 2001;19:305-13.
13. Jiao X, Krasna MJ, Sonett J, et al. Pretreatment surgical lymph node staging predicts results of trimodality therapy in esophageal cancer. Eur J Cardiothorac Surg. 2001;19:880-6.

14. Siewert RJ, Feith M, Werner M, Stein HJ. Adenocarcinoma of the esophagogastric junction: results of surgical therapy based on anatomical/topographic classification in 1,002 consecutive patients. Ann Surg. 2000;232:353-61.

15. Izbicki JR, Hosch SB, Pichlmeier U, et al. Prognostic value of immunohistochemically identifiable tumor cells in lymph nodes of patients with completely resected esophageal cancer. $N$ Engl J Med. 1997;337: 1188-94.

\section{JTCVS On-Line Manuscript Submission and Review}

\section{Please visit http://www.editorialmanager.com/jtcvs/}

Effective September 15, 2001, authors and reviewers may submit manuscripts and reviews electronically via Editorial Manager, our new Web-based system with full electronic submission, review, and status update capabilities.

As we move from paper to electronic submissions, the Editorial Office will make proxy submissions of all manuscripts accompanied by a diskette containing the electronic files of the text, tables, and figures. Editors, authors, and reviewers will receive automatic e-mails when significant events occur.

We strongly encourage all authors and reviewers to use Editorial Manager. Although we will continue to accommodate the submission of paper manuscripts for some months, our goal is to be completely electronic within 9 to 12 months.

All individuals currently in our database for whom we have e-mail addresses will receive via e-mail a system-assigned username and password that can be used to log in to the system without prior registration. All those not receiving the e-mail must register the first time they use the system.

As with any broad systemic change, the conversion to the new system will take some time to complete. We ask your patience as we replace our in-office database with the new system. We also encourage you to take advantage of the speed and efficiency that the new system will provide for us all: editor, author, reviewer, and publisher. 